\title{
Constructivismo social en la pedagogía
}

\author{
José Esteban Pinto Ladino * \\ Victor Alonso Castro Bello ** \\ Orfa Mireya Siachoque Castillo ***
}

Artículo de reflexión

Fecha de Recepción: 6 noviembre 2016.

Fecha de Aprobación: 8 abril 2017.

\section{Resumen}

El artículo se aborda desde la investigación científica y tecnológica, de los resultados obtenidos al examinar la apropiación pedagógica del constructivismo social en la práctica docente del área de matemáticas en la Institución Educativa Magdalena de Sogamoso (Boyacá). La metodología utilizada para el estudio es de tipo cualitativo, con enfoque crítico social, así mismo la investigación-acción, mediada por una intervención de clase constructivista para dar respuesta a las necesidades detectadas en el área de matemáticas en los estudiantes de séptimo, octavo y noveno. Como resultado de la investigación, se evidenció que las prácticas docentes tienen influencias del modelo tradicionalista y tecnológico para la enseñanza de las matemáticas. Con respecto al desarrollo de las clases constructivistas, se identificó que superaron los niveles insuficientes a suficientes; en cuanto a interacción docente-estudiante, ambiente de aula, normas de convivencia, no obstante, las estrategias metodológicas requieren un cambio en la selección concreta de los recursos disponibles, la motivación, la propuesta de situaciones reales para propiciar el aprendizaje constructivo y favorecer el desarrollo de destrezas desde las matemáticas para la vida.

Palabras clave: constructivismo, tendencias pedagógicas, currículo, Proyecto Educativo Institucional.
* Institución Educativa Magdalena, Sogamoso (Boyacá-Colombia) jepint2@gmail.com ** Institución Educativa Magdalena, Sogamoso (Boyacá-Colombia) vialcabe1972@gmail.com

*** Institución Educativa Magdalena, Sogamoso (Boyacá-Colombia) yeyasiachoque@gmail.com

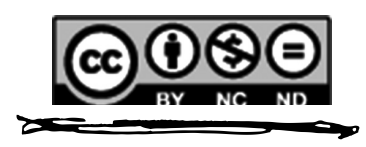




\section{Introducción}

La educación contemporánea enfrenta problemas de diferentes tipos, que se han generado por los cambios abruptos que convergen desde diferentes esferas. Al respecto, Esteve (2004) propone que, para transformar la enseñanza, se deben tener en cuenta factores fundamentales como la cultura y las condiciones de trabajo de los profesores por su determinado contexto histórico. Lo anterior se debe, en primer lugar, al contexto macro que depende de la evolución de las fuerzas sociales (política y economía); también, en segundo lugar, a las leyes y decretos con capacidad de cambio limitado; y, finalmente, a la práctica de los docentes por ser quienes, en últimas, tienen su propia mentalidad de enseñar.

Dentro de los nuevos horizontes de la educación, el Ministerio de Educación Nacional de Colombia ha promovido evaluaciones anuales mediante las pruebas SABER y el Índice Sintético de Calidad, con el objetivo de consolidar un punto de partida para poner en marcha a las instituciones educativas en conocer cómo se encuentran los estudiantes frente a algunas competencias del saber, saber hacer y ser, desde la perspectiva de ofrecer una calidad en la educación. Es así que, estas pruebas permiten no solo medir cuánto han aprendido los estudiantes, sino también evaluar si el docente facilita el saber y en qué grado se ha desarrollado en la práctica docente siguiendo los lineamientos curriculares.

La investigación surge del análisis de los resultados de la prueba SABER aplicada en los grados 3 y 5 de primaria, y 9 y 11 de secundaria de la Institución La Magdalena, donde se halló en la descripción general de matemáticas del grado noveno que el $82 \%$ de los estudiantes no establecen relaciones entre propiedades de gráficas y ecuaciones algebraicas, el $75 \%$ no identifican relaciones entre diferentes unidades utilizadas para medir cantidades de la misma magnitud y determinar su pertinencia, el $75 \%$ no reconocen las posibilidades de ocurrencia de un evento a partir de los datos y, por último, el 72 $\%$ de los estudiantes no reconocen el lenguaje algebraico como una forma de representar procesos inductivos (ICFES, 2016, p. 14).

Fue a partir de esta valoración de las pruebas Saber, que se inicia la búsqueda delas causas por las cuales los estudiantes de grado noveno tienen dificultades en el área de matemáticas, por tal motivo, se suscitaron interrogantes desde la práctica docente como: ¿cuáles son las necesidades de los estudiantes?, ¿qué modelo pedagógico puede dar respuesta a las necesidades de los estudiantes?, ¿cuáles son las realidades de las prácticas docentes en el aula?, ¿qué percepción tiene el estudiante de la práctica docente? en muchas otras, ubicando la que ocupa la investigación ¿cómo mejorar la enseñanza de las matemáticas desde la apropiación del modelo constructivista en la práctica docente y facilitar el aprendizaje en los grados de séptimo a noveno?

En este sentido, la investigación se enmarcó dentro de la metodología de tipo cualitativo, con enfoque socio- 
crítico, tomando como punto de referencia la actuación del docente en la práctica pedagógica con base en el modelo constructivista social y la percepción de los estudiantes frente al desarrollo de este modelo en el aula de clase; e implicó abordar la investigaciónacción, propuesta por Elliot (1993) a través de las fases de planeación, acción y evaluación. Para la recolección de la información, se utilizó una encuesta a estudiantes para hallar la percepción de la práctica docente, una encuesta para docentes con base en Porlán (1995), y adaptada de acuerdo con los modelos pedagógicos tradicionalista, tecnológico, espontaneista y constructivista, una entrevista semiestructurada para los docentes del área de matemáticas.

\section{Retrospectiva de investigación}

A nivel internacional, los autores Gómez y Perdomo (2015) presentan una investigación sobre el modelo constructivista propuesto por Piaget (1971), relacionado con la práctica docente, la importancia del modelo estipulado en el Proyecto Educativo Institucional, las concepciones del profesor que orienta el comportamiento de un sujeto, desde los aspectos conductual, humanístico y cognoscente. De igual manera, se abordaron temas como: ambientes de aprendizaje y la renuencia al cambio por parte de algunos docentes. En los resultados del estudio, se confirmó que los docentes hacen uso deficiente de una planeación que guíe sus prácticas de aula y, por consiguiente, no existen metas tangibles de aprendizaje para los estudiantes.

La importancia de unificar y conocer el modelo constructivista establecido desde el PEI de la Institución, tiene relación estrecha con la práctica docente en el aula, pues desde allí es de donde parten las directrices metodológicas para lograr un aprendizaje en función de adquirir destrezas para desenvolverse ante los fenómenos cognitivos y sociales.

Por otro lado, en el estudio realizado por Castillo (2008), se propone aplicar el modelo constructivista para la enseñanza de las matemáticas, incorporando las tecnologías de la información y comunicación. El programa educativo se desarrolló a través de talleres durante seis meses, fue evaluado comparando el rendimiento académico antes y después de la intervención. Los resultados permitieron concluir que, el diseño de estrategias constructivistas apoyadas por la tecnología, son apropiadas para transformar la práctica docente y el aprendizaje de las matemáticas.

A nivel nacional, la investigación realizada por Nieto (2008) se centra en el desarrollo de proyectos matemáticos, con base en cuatro elementos que corresponden a los modelos pedagógicos: Espontaneista, Tradicional, Tecnológico y Constructivista, cada uno de ellos con diez alternativas de respuestas distribuidas de forma aleatoria. Este estudio demostró que, estadísticamente, el pensamiento pedagógico de los docentes es constructivista; no obstante, en la realidad del aula, la práctica docente se desarrolla tomando metodologías de
Los autores Gómez y Perdomo (2015) presentan una investigación sobre el modelo constructivista propuesto por Piaget (1971), relacionado con la práctica docente, la importancia del modelo estipulado en el Proyecto Educativo Institucional, las concepciones del profesor que orienta el comportamiento de un sujeto, desde los aspectos conductual, humanístico y cognoscente. 
varios modelos pedagógicos y los adapta a su conveniencia.

\section{Fundamento del conocimiento teórico}

El Ministerio de Educación Nacional -MEN (2008) define el Proyecto Educativo Institucional (PEI), como la carta de navegación de las escuelas y colegios, en donde se especifican, entre otros aspectos, los principios y fines del establecimiento, los recursos docentes y didácticos disponibles y necesarios para ofrecer a la comunidad educativa las estrategias metodológicas y administrativas con las que cuenta la institución para atender a los estudiantes. Según el artículo 14 del Decreto 1860 de 1994, toda institución educativa debe elaborar y poner en práctica con la participación de la comunidad, un proyecto educativo institucional que exprese la forma como se ha decidido alcanzar los fines de la educación definidos por la ley, teniendo en cuenta las condiciones sociales, económicas y culturales de su medio. El proyecto educativo institucional debe responder a situaciones y necesidades de los educandos, de la comunidad local, de la región y del país, ser concreto, factible y evaluable (MEN, 2006).

El modelo pedagógico, según Posner (2005), se define como "una herramienta conceptual inventada por el hombre para establecer mejor algún evento; un modelo es la representación del conjunto de relaciones que describen un fenómeno",
Por otra parte, el PEI cuenta con cuatro componentes: Gestión directiva y horizonte institucional, Gestión administrativa y de talento humano, Gestión de la comunidad y Gestión pedagógica (MEN, 2008, p. 8).

El componente Pedagógico es la razón del proceso enseñanza-aprendizaje, es el eje central alrededor del cual giran los demás componentes y en este se plantea el currículo de la institución educativa, que servirá como guía para orientar la práctica docente; allí, se especifican las intenciones educativas como los planes de acción apropiados para alcanzar los propósitos planteados, y el modelo pedagógico oportuno de acuerdo con el contexto de la población.

El modelo pedagógico, según Posner (2005), se define como "una herramienta conceptual inventada por el hombre para establecer mejor algún evento; un modelo es la representación del conjunto de relaciones que describen un fenómeno", (p.24). Un modelo pedagógico es una representación de las relaciones que predominan en el fenómeno de enseñar. Un modelo pedagógico, como representación de una perspectiva pedagógica, es también un paradigma que puede coexistir con otros paradigmas dentro de la pedagogía y que organiza la búsqueda de los investigadores hacia nuevos conocimientos en el campo.

De lo anterior, se infiere que un modelo pedagógico es la forma de orientar los procesos formativos de una institución educativa, buscando una formación integral (Nova, 2015), por tanto, es la base sobre la que se cimientan todas las estrategias que deben abarcar los procesos de enseñanza-aprendizaje, para formar al hombre que se quiere educar.

Según Flórez (1994), el modelo pedagógico es concebido como:

[...] más allá de los llamados fundamentos y ciencias de la educación, 
cuya dispersión ha repercutido naturalmente en una aproximación escondida sobre la práctica educativa, es necesario confrontar teoría y práctica con una estructura más fundamental, subyacente a todo pensar y actuar educativo, que actúa como supuesto de todo modelo y estrategia pedagógica particular. (p. 114).

De lo anterior, se deduce que el modelo pedagógico se fundamenta en una teoría para pensar y actuar en la práctica educativa, por consiguiente, el conocimiento del modelo pedagógico por parte de los docentes es un factor importante para generar cambios no solo en el aula de un docente sino un cambio institucional, que beneficie a la comunidad.

Los Modelos Pedagógicos son las representaciones de las corrientes pedagógicas que coexisten como paradigmas dentro del campo disciplinario de la pedagogía. El modelo es un instrumento analítico para describir, organizar y entender la multiplicidad, la diversidad, contingencias y estructuras que presenta una corriente pedagógica (Bernal, 2004, p. 102).

Debido a las demandas políticas, económicas y sociales, los modelos pedagógicos son concebidos y desarrollados a través del tiempo, de esta manera, se marcan las tendencias con base en las necesidades de producción, algunas de ellas son:

Posner(2005). Tradicional, experiencial, disciplinar, conductista y cognitivo.
Flórez (1994). Tradicional, conductista, constructivista y social.

Porlán (2002). Tradicionalista, conductista, tecnológico, espontaneista y constructivista.

Ortiz (2011) hace una nueva clasificación de los modelos pedagógicos en cuatro grandes grupos: la pedagogía tradicional; la escuela nueva; la tecnología educativa, la escuela del desarrollo integral; y, finalmente, se propone una alternativa emergente, tanto para la investigación científica como para la educación. Así como también el paradigma científico neuroconfiguracional, para hacer ciencia humana y social.

El constructivismo social como modelo pedagógico, según Vygotsky (1978), considera que el desarrollo humano es un proceso de desarrollo cultural, siendo la actividad del hombre el motor del proceso de desarrollo humano. El concepto de actividad adquiere, de este modo, un papel especialmente relevante en su teoría social. Para él, el proceso de formación de las funciones psicológicas superiores, se dará a través de la actividad práctica e instrumental, pero no individual, sino en la interacción o cooperación social.

En este sentido, las funciones mentales superiores para Vygotsly (1978), "son las que se adquieren y se desarrollan a través de la interacción social” (p. 5). Es decir, las que el individuo aprende en el contexto donde se desenvuelva, pues allí se determina específicamente su manera de pensar, actuar, aprender y socializar.
Los Modelos Pedagógicos son las representaciones de las corrientes pedagógicas que coexisten como paradigmas dentro del campo disciplinario de la pedagogía. 
Según Ñeco (1999), la función del docente en el constructivismo se distingue por las características del modelo por competencia (saber hacer), metodología activa dirigida hacia la colaboración y la cooperación, guía y controla la actividad del niño, interactúa con el estudiante en la formación del ser como persona en un contexto social específico que evalúa y retroalimenta.

Así mismo, afirma que para el desarrollo de la clase, desde la perspectiva constructivista, se debe tener en cuenta: el contexto del aula (ambiente físico y social), conocimientos previos de los estudiantes, especificar con claridad los propósitos de la clase, ubicar con certeza a los estudiantes en el grupo (trabajo colaborativo), explicar claramente la tarea a realizar y la estructura del fin, monitorear la efectividad del grupo que atiende y evaluar continuamente el nivel de logros de todos los estudiantes.

\section{Metodología}

La metodología desarrollada fue de carácter cualitativa, con enfoque investigación-acción y de tipo descriptivo. Según Elliot (2000), la investigación acción (IA) conjuga la exploración, la actuación y la valoración de los resultados.

Fase de planeación: en primera instancia, la identificación de la problemática, con base en el análisis de los resultados de las pruebas SABER (2016), donde se detectó el bajo rendimiento en el área de matemáticas.
Para el cumplimiento del primer objetivo específico, con respecto a la identificación de las posturas pedagógicas de los docentes de la Institución Educativa Magdalena en el aula, se utilizó tres instrumentos de recopilación de la información, los cuales fueron:

- Encuesta adoptada para la investigación de acuerdo con los modelos pedagógicos tradicionalista, tecnológico, espontaneista y constructivista, a todos los docentes de la Institución en un total de 30.

- Entrevista semiestructurada, aplicada a cuatro docentes del área de matemáticas, utilizando como punto de partida el modelo constructivista y avaladas por el experto José Francisco Leguizamón, director del presente proyecto. Con la aplicación de esta herramienta, se identificó las concepciones de los docentes.

- Encuesta a estudiantes. El objetivo de la encuesta para estudiantes, fue el de identificar cómo percibe la práctica del docente en la clase antes de la intervención con los talleres diseñados con base en el modelo constructivista, para compararla en la fase de evaluación.

Fase de Acción. En esta fase, se desarrolló el segundo objetivo específico, con respecto a verificar las prácticas desarrolladas por los docentes de matemáticas en el aula, mediadas por talleres matemáticos diseñados con fundamentos en el modelo constructivista. 
En la recolección de la información, se utilizó la observación de clase desarrollada a través de tres talleres aplicados a los estudiantes:

- Observación de clase. Las observaciones de clase se realizaron a los cuatro docentes del área de matemáticas, en un primer momento, antes del desarrollo de talleres; y, en un segundo momento, durante el desarrollo de los tres talleres construidos bajo el modelo constructivista.

- Taller de aula. El taller fue diseñado sobre tres aspectos: planeación, desarrollo y evaluación, factores que tiene en cuenta el modelo constructivista para el desarrollo de la práctica docente, se tuvo en cuenta los aspectos de ambiente en el aula, normas de convivencia, entorno de aprendizaje, estrategias metodológicas y evaluación.

Recogidos los datos a través de la entrevista, encuesta y la observación, se categorizó la información para el análisis. Según Corbetta (2003), las categorías se establecen de forma inductiva, como ocurre en toda investigación cualitativa.

Tabla 1. Categorización de la información

\begin{tabular}{|c|c|c|}
\hline Categoría & subcategoría & subcategorías \\
\hline \multirow{14}{*}{$\begin{array}{c}\text { MODELO } \\
\text { PEDAGÓGICO } \\
\text { CONSTRUCTIVISTA }\end{array}$} & \multirow{6}{*}{ Planeación } & Tiempo \\
\hline & & Adaptación a las necesidades escolares \\
\hline & & Planeación estratégica \\
\hline & & Planeación de evaluación \\
\hline & & Interacción docente-estudiante \\
\hline & & Utilización del espacio \\
\hline & \multirow[t]{5}{*}{ Desarrollo } & Ambiente del aula \\
\hline & & Normas de convivencia \\
\hline & & Entorno para el aprendizaje \\
\hline & & Interacción docente-estudiante \\
\hline & & Estrategias metodológicas \\
\hline & \multirow[t]{3}{*}{ Evaluación } & Estrategias de evaluación integral \\
\hline & & Seguimiento a procesos \\
\hline & & Ajustes y retroalimentación \\
\hline
\end{tabular}

Fuente: creada autores

\section{Resultados}

A continuación, se presenta el análisis de la información obtenida a partir de la encuesta y entrevista semiestructurada a los docentes.

\section{Encuesta (Tendencias pedagógicas de la IEM)}

Una vez aplicado el primer instrumento de investigación (Tendencias pedagógicas presentes en los docentes de 


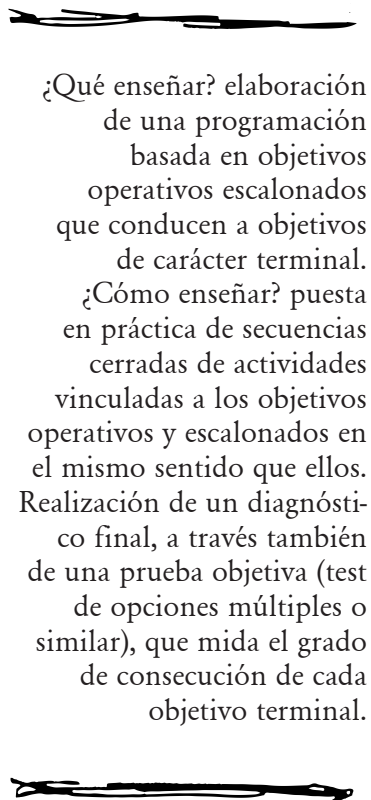

la IEM), se procede a realizar el análisis de acuerdo con la clasificación de las tendencias contempladas por Porlán, de donde se obtuvieron las siguientes reflexiones.

Tendencia tradicional. El docente de la IEM aún considera que el orden del salón de clase es fundamental para el buen desarrollo de la misma, partiendo de la ubicación de los estudiantes que deben estar dispuestos de tal manera, que solo observen al docente, donde el silencio y la disciplina son factores fundamentales para lograr un buen resultado académico. El docente se convierte en un prototipo dominante quien dice quién opina y quién no, evitando controversias que obstaculicen el desarrollo normal de la clase, siendo estos rasgos característicos del pensamiento pedagógico tradicional. Como lo determina Porlán (1996),

Las conductas de aquellos alumnos que demuestran una falta de conexión con el hilo conductor de la clase, cuando no hay una clara interferencia con el esquema previsto (alumnos distraídos, contestaciones fuera de contexto, relaciones paralelas entre alumnos, etc.), son una expresión de la tendencia que poseen hacia la indisciplina y la Inadaptación escolar. Por el contrario, las conductas de aquellos alumnos que externamente demuestran atención y toman apuntes, son un reflejo fiel de que están siguiendo comprensivamente la explicación del profesor. (p. 27).

Tendencia tecnológica. De esta tendencia se puede interpretar que los docentes de la IEM tienen una fuerte influencia tecnológica, puesto que sus clases están orientadas al cumplimiento de objetivos, que son trazados por los derechos básicos de aprendizaje y/o los ejes temáticos de las áreas fundamentales, lo cual condiciona, en una buena parte, las prácticas docentes. De las diez preguntas planteadas en la encuesta, siete tuvieron aceptación.

Lo anterior permite inferir que los docentes están permeados en un alto grado por esta concepción pedagógica, donde se plantea la evaluación dirigida a la persecución de un logro ordenado de manera jerárquica, que fuerza a la uniformidad y favorece el aprendizaje mecánico e instrumental, preparando al estudiante para responder pruebas externas, sin valorar sus propias opiniones, que son las pautas de actuación características de la tendencia tecnológica. Según Porlán (1995),

¿Qué enseñar? elaboración de una programación basada en objetivos operativos escalonados que conducen a objetivos de carácter terminal. ¿Cómo enseñar? puesta en práctica de secuencias cerradas de actividades vinculadas a los objetivos operativos y escalonados en el mismo sentido que ellos. Realización de un diagnóstico final, a través también de una prueba objetiva (test de opciones múltiples o similar), que mida el grado de consecución de cada objetivo terminal. (p. 32).

Entender el aprendizaje en una sola línea de acción irreversible, impide interpretar los procesos que manifiesta cada estudiante. 
Tendencia espontaneista. De esta tendencia, se evidencia que los docentes están medianamente permeados, donde priman rasgos como la importancia de la motivación en el estudiante, sus intereses y privilegia el contexto cultural, social y económico, por encima de los contenidos, los objetivos y la evaluación.

El estudiante se educa en vinculación permanente con la realidad que lo rodea, desde la filosofía de que el aprendizaje verdadero se da como resultado de sus intereses y experiencias, y se halla en el entorno en que vive, se pretende despertar en el estudiante la observación y el descubrimiento, dejando de lado el conocimiento desde los contenidos supuestamente importantes en cada área. Desde esta tendencia, el docente prepara los contenidos teniendo en cuenta el escenario cultural en el que se evidencia el significado y la respuesta, que a su vez sirve de base para estimular la nueva pregunta, lo cual seguirá permitiendo la generación de otras nuevas preguntas que nacen del proceso mismo del estudiante.

Este proceso es una interacción activa entre el docente y el estudiante con una acomodación entre ambos. Porlán (1995) plantea que "la enseñanza para que provoque realmente aprendizaje debe basarse fundamentalmente en las motivaciones espontáneas de los estudiantes. Modificación y readecuación permanente del plan de trabajo en función de los intereses y la motivación de los alumnos, utilizando estrategias de improvisación durante la misma acción”. (p. 41).
Tendencia constructivista. Los docentes están de acuerdo con ocho de las diez preguntas que apuntan al constructivismo, es decir, consideran importante que el conocimiento sea un proceso individual, depende de los pre saberes, se aprende a partir de la problematización de lo que supuestamente ya se sabe, nunca el estudiante llega con una concepción vacía acerca de un concepto, aprender es un proceso que no se realiza por transmisión directa, el profesor es un generador de ambientes de aprendizaje, la interacción entre estudiantes y estudiante-docente son básicos para lograr aprendizajes significativos, el reconocimiento de las necesidades es fundamental para lograr aprendizajes, se deben tener en cuenta los diferentes estilos cognitivos de los estudiantes.

El proceso de evaluación que se lleva a cabo solo se dedica a verificar objetivos previamente estipulados en el plan de área.

En dos preguntas que pretendían identificar lo mismo (la importancia de la problematización del pre saber), se observó contradicción, es decir, resultados encontrados. La práctica pedagógica del docente se está desarrollando en torno a una guía sin tener en cuenta los pre saberes que tiene el estudiante de su contexto, no considera relevante el enlace entre la estrategia para promover los conocimientos previos y la nueva información. Díaz y Hernández (1999) afirman que la articulación de los conocimientos previos y la nueva información potencian los aprendizajes significativos. 
El pensamiento del docente, en su gran mayoría, es constructivista, pero está influenciado por varias tendencias pedagógicas, primando la tecnológica desde la planeación de su clase, fijada en el cumplimiento de objetivos y metas para garantizar la enseñanza eficaz, observable y cuantificable en el desempeño académico del estudiante; se evidenció que los docentes utilizan, en menor medida y al mismo nivel, la tendencia espontaneísta y tradicional, por considerar un factor preponderante los intereses del estudiante, desarrollando el proceso de enseñanza desde su contexto sociocultural por encima de los contenidos, los objetivos y la evaluación. En la tendencia tradicional, el profesor es considerado como un prototipo de autoridad, siendo un factor importante para conservar la disciplina, el orden y disposición de los estudiantes en el salón de clase.

\section{Entrevista semiestructurada}

Se realizó la entrevista a los docentes a través de cinco preguntas. En el análisis de las respuestas, se encontró:

$¿$ Usted considera que los conocimientos previos que posee el estudiante sobre el tema son un obstáculo para el aprendizaje?

Todos los docentes coinciden en que los conocimientos previos del estudiante son importantes porque todo conocimiento es construido con base en la experiencia, lo que lleva a una transformación de las existentes. Es decir que, constantemente el que aprende está construyendo su propio conocimiento.
Los entrevistados coinciden en la importancia de los pre saberes y algunos van un poco más allá, notándose la tendencia constructivista, en donde el andamiaje lo comparan con una telaraña, como lo expresa el docente D5 "No, yo creo que es al contrario, un buen aprendizaje debe basarse siempre en los conocimientos previos que son la base para que los estudiantes articulen los nuevos conocimientos que tienen, es como una especie de telaraña, haciendo la analogía, para que ellos de esa manera vayan construyendo su conocimiento sobre la base conceptual que ellos ya poseen, con base en las experiencias de vida que ellos ya han tenido".

La respuesta anterior guarda estrecha relación con lo expresado por Posner (2005), "la meta educativa es que cada individuo acceda progresiva y secuencialmente a la etapa superior de su desarrollo intelectual de acuerdo con las necesidades y condiciones de cada uno" (p. 25).

Porlán (1995) afirma que "desde un punto de vista educativo, se trabaja desde y para el conocimiento que tienen, generan y construyen los alumnos" ( $\mathrm{p}$. 105). La postura de Porlán se evidencia en lo manifestado por el docente D22 "los obstáculos son importantes, son básicos para que el estudiante pueda entender los temas que se tratan en las clases, obviamente que él tiene que relacionar los conceptos previos con lo nuevo que ve en clase, para poder realmente tener un aprendizaje significativo".

$¿$ El docente debe incentivar al estudiante para que utilice diferentes formas de 
representación de los problemas o situaciones para comprenderlos?

Todos los docentes están de acuerdo con la importancia que tiene el hecho de que el estudiante tenga distintos puntos de vista frente a una situación determinada, en sus afirmaciones tienen en cuenta los diferentes métodos de aprendizaje de los estudiantes (inteligencias múltiples), una inteligencia implica la habilidad necesaria para resolver un problema o para elaborar productos que son importantes en un contexto cultural (Gardner, 1995). De las respuestas obtenidas por los docentes, la más afín con lo expresado por Gardner, fue manifestada por el docente:

D18 “A mí me parece que es importantísimo que los problemas se vean desde diferentes puntos de vista, si nosotros nos quedamos con una sola información acerca de un asunto, se va a cerrar la posibilidad de que el cerebro busque muchas soluciones, si lo vemos desde diferentes enfoques o desde diferentes temáticas, el mismo cerebro va a ayudar a que el estudiante aprenda que todos los problemas que se le presentan en la vida tienen diferentes soluciones $y$ ayudan a que en su aprender académico tengan más responsabilidad y en sus problemas en la vida tengan amplitud para poderlos solucionar y así no acudan a las soluciones más rápidas".

¿La enseñanza a partir de problemas de la vida cotidiana, sirve especialmente para desarrollar en el estudiante el concepto?

Todos los docentes coinciden en la importancia de la enseñanza a partir de problemas de la vida cotidiana, ya que esto motiva al estudiante en el aprendizaje, partiendo de una realidad conocida por él, lo cual permite una interiorización que genera una reestructuración interna del pre saber para modelar el nuevo concepto.

Esto pone en manifiesto que los docentes de la IEM, tienen una concepción mezclada entre rasgos tecnológicos y progresistas, ya que todos coinciden en afirmar que el aprendizaje se hace efectivo a partir de problemas de la vida cotidiana, como lo manifiesta:

D15 "Claro, es que es mejor aprender con algo que uno ha vivido desde la experiencia, es mejor aprender que dos manzanas más dos manzanas teniéndolas al frente, son cuatro y no simplemente dos números en el tablero, porque al saber un concepto a partir de la experiencia se graba en el corazón, en la mente y en el alma, entonces no solamente se tiene el concepto en la mente sino que se puede poner en práctica en todo, que es lo que debería ser la educación dar herramientas para la vida personal, académica, social". Posner (2005) propone que "la meta educativa es que cada individuo acceda, progresiva y secuencialmente a la etapa superior de su desarrollo intelectual de acuerdo con la necesidades y condiciones de cada uno" (p. 25).

¿Los estudiantes aprenden fuera de la escuela aspectos sobre el tema, pero el aprendizaje real se da en la escuela?

A pesar de que todos los docentes no estén totalmente de acuerdo, sí 
"El aprendizaje en el aula es global, porque fuera del aula se procesan otras enseñanzas que, de cierta manera, influyen en lo cognitivo (Pinedo \& Yañez, 2017), por ello no es sesgado, sino más bien compartido, no se da solamente con un cuaderno, un libro y un lápiz. Este esencialmente se da en

las experiencias de todos

los entornos de la vida, si nosotros educáramos para la vida, cambiaría radicalmente la sociedad". se evidencia la importancia que el docente le da al aprendizaje fuera del aula, teniendo en cuenta la teoría constructivista social (Vygotskiana), que propone que el primer aprendizaje real comienza a darse de manera social en el medio donde se desarrolla una persona y en el momento en que comienza a aparecer el lenguaje.

Apreciaciones importantes alrededor del aprendizaje, son expresadas por el docente:

D4 "El aprendizaje en el aula es global, porque fuera del aula se procesan otras enseñanzas que, de cierta manera, influyen en lo cognitivo (Pinedo \& Yañez, 2017), por ello no es sesgado, sino más bien compartido, no se da solamente con un cuaderno, un libro y un lápiz. Este esencialmente se da en las experiencias de todos los entornos de la vida, si nosotros educáramos para la vida, cambiaría radicalmente la sociedad".

¿Trabajar la temática de forma individual es la mejor estrategia que puede utilizar el docente, ya que si se permite que los estudiantes discutan o se comuniquen entre ellos, se pierde el tiempo y no se obtienen rendimientos?

Se evidencia que para todos los docentes es relevante y de gran importancia el trabajo colaborativo, el cual ha sido aplicado por todos ellos, pero además este trabajo en algunos casos no ha sido tan exitoso, como lo manifiesta:

D5 "Yo pienso que el trabajo en equipo, no tanto en grupo, sino en equipo, es bien importante, siempre les recalco a ellos la importancia de la diferencia entre el trabajo en grupo y el trabajo en equipo, el trabajo en equipo es donde todos trabajan, todos aportan, diferente del trabajo en grupo que unos se recuestan en el trabajo de los otros y no tienen una participación activa; yo pienso que la escuela debe fomentar el trabajo en equipo, en esa forma, para que ellos hagan intercambio, discutan, debatan y sobre esa base ellos lleguen a acuerdos, a conclusiones que sean favorables para todo el equipo y para la sociedad también".

El trabajo colaborativo es un punto álgido en el aprendizaje constructivista, ya que es enriquecedor principalmente por el debate, el cual desarrolla en el estudiante la parte reflexiva y social, donde el estudiante conoce las ideas de sus compañeros, reflexiona sobre sus errores, para así entender que nadie tiene la verdad absoluta y que el proceso de aprendizaje es un proceso reflexivo y tolerante ante las diferencias de opinión. Porlán (1995) plantea que conseguir compaginar una participación espontánea con una dirección no autoritaria del proceso de aprendizaje, debe ser una característica del docente constructivista.

Del análisis de los resultados obtenidos en el desarrollo del primer objetivo específico de esta investigación, se concluye que los docentes de la IEM estáninfluenciados porvariastendencias pedagógicas, evidenciándose fortalezas como: importancia de los presaberes, la enseñanza a partir de problemas, el trabajo colaborativo, contextualización y se tiene en cuenta la opinión de los 
estudiantes, siendo estas características del constructivismo social. Es de notar que, para los docentes de la Institución no se le da la debida importancia a la reestructuración del concepto.

\section{Práctica del docente con el diseño de talleres constructivistas}

Para el cumplimiento de la fase de acción, se aplicaron tres talleres a los estudiantes, estos fueron valorados a través de una observación a la práctica del docente en el salón de clase; una encuesta a estudiantes; y el rendimiento académico alcanzado por los educandos del área de matemáticas. Los resultados obtenidos permitieron evidenciar lo siguiente:

En un primer acercamiento a la práctica docente en el aula, se encontró que la mayoría de los docentes emplea un orden sistemático para el planeamiento de clase la mayor debilidad en el planeamiento de la interacción con el estudiante, metodologías y la utilización de los recursos disponibles, lo que indica que la falta de indagación con base en estrategias constructivistas los limita a la hora de planear la clase, y las actividades rutinarias pueden generar la monotonía en la percepción del estudiante. Por tal motivo, es necesario que realicen cambios significativos al abordar la planeación con respecto a las estrategias metodológicas orientadas hacia la perspectiva constructivista, que de acuerdo con Olson (1982), "desde el cambio en los puntos de vista de los docentes del proceso enseñanza-aprendizaje debe buscar escudriñar de la manera más sencilla acciones metodológicas" (p. 4). En este sentido, estar actualizando permanentemente para mejorar día a día su práctica y lograr sus propósitos con cada estudiante, teniendo en cuenta el currículo como eje fundamental de su quehacer pedagógico y las necesidades e intereses de los estudiantes.

En un segundo momento de observación, se constató que los docentes replantearon la metodología de la forma como la construcción del conocimiento en el aula va más allá de la relación del profesor y estudiantes, hacia una amplia interacción entre ellos mismos en el entorno social y cultural de la clase, llevándola al plano de la resolución de nuevos hechos matemáticos apoyándose en la experiencia que ya poseen los educandos, de esta forma, facilitar la comprensión mayor de cómo y cuándo aplicar las operaciones matemáticas y cómo adaptarla a nuevas situaciones.

De acuerdo con los resultados de la comparación de las observaciones realizadas de las prácticas docentes, en un primer momento, antes de aplicar los talleres y durante la aplicación de los talleres, se puede concluir para el Desarrollo de clase, que:

El ambiente de aula mejoró de un nivel insuficiente a suficiente, lo que significó que se tuvo en cuenta los aspectos de saludar a los estudiantes, rechazar comportamientos inadecuados y tratar a todos los estudiantes por igual.

Para el aprendizaje: la valoración fue muy suficiente, lo que reflejó un "desde el cambio en los puntos de vista de los docentes del proceso enseñanza-aprendizaje debe buscar escudriñar de la manera más sencilla acciones metodológicas" 
mayor planeamiento en utilizar los recursos disponibles de la institución para la enseñanza, facilitando a los estudiantes una aproximación a escenarios apropiados para el proceso de comunicación, comprender qué sentido tienen la matemáticas en la vida cotidiana, cómo pueden aplicarlas en la vida real, obtener la información de objetos con los que tienen contacto todos los días. Así como también la recursividad como estrategia para superar las dificultades, producto de vacíos esencialmente causados por el desinterés e irresponsabilidad en las tareas de matemáticas.

La normatividad de convivencia: se logró afianzar la importancia de la disciplina, el respeto, el ambiente de aula, a través de la resolución de problemas matemáticos traspuestos al plano de los conflictos, lo que aportó de manera significativa a la utilidad de las matemáticas no solo en el sistema numérico, sino también en el aspecto social. Por otro lado, los docentes aplicaron el constructivismo para acercar al estudiante hacia el aprendizaje con una mayor percepción en la dimensión afectiva y cognitiva positiva hacia las matemáticas.

En la interacción docente-estudiante: se logró fortalecer la comunicación a un nivel suficiente, superando algunas barreras que los distanciaban, como la confianza respetuosa, lo que le exigió al docente una gran capacidad de aceptación, al estudiante el respeto por la opinión del otro, para confrontar, concertar para solucionar problemas y mantener actitudes de reciprocidad, dinámicas que permitieron mejorar la disposición para las matemáticas, sin miedos al fracaso y aprendiendo de los errores. Por otro lado, el docente de modelo tradicionalista asumió el papel activo del estudiante, reconociendo que el modelo constructivista social corresponde con un mundo socialmente construido, que crea la experiencia compartida de la realidad física, que reside de una modificación constante. De acuerdo con Piaget (1993) y Londoño (2016), el constructivismo social considera al sujeto individual y el dominio de lo social como indisolublemente interconectados, en otras palabras, se compone de la interacción social donde se generan procesos individuales que se dinamizan en contextos participativos.

Estrategias metodológicas: este aspecto fue considerado como uno de los más importantes, puesto que se avanzó de un nivel insuficiente a suficiente, lo que evidenció un cambio de parte del docente en la propuesta de las situaciones didácticas en las actividades para el aprendizaje de las matemáticas, lo que implicó un replanteamiento en el modelo pedagógico más concreto y definido para el uso de las estrategias adecuadas para la enseñanza y propiciar la construcción del conocimiento matemático desde el contexto en el que se desenvuelven los educandos, apreciando sus experiencias y satisfacer sus necesidades cognitivas, afectivas y sociales a través de la adquisición de destrezas para solucionar problemas a nivel individual $y$ en equipo, aproximándolo a vivir verdaderas experiencias que lo abastecen de 
materia prima para las matemáticas desde una perspectiva social.

La evaluación también tuvo un cambio relevante, pasó del nivel insuficiente a suficiente, lo que significó una mejor valoración de las actividades encausadas a medir el conocimiento construido y retroalimentar al estudiante con mayor énfasis en las dudas y procedimientos de los ejercicios para alcanzar los objetivos planteados. Aún queda un aspecto por superar como el de hacer comentarios sobre el buen desempeño y asegurar que los estudiantes se retroalimentaran de los errores para visibilizar las barreras en la comprensión de las matemáticas y superaran las dificultades, así como también el monitoreo continuo en el aula de clase de la efectividad de los resultados de las actividades.

\section{Conclusiones}

Las tendencias pedagógicas que se evidencian en las prácticas docentes, se orientan hacia el tradicionalismo con un $33 \%$, al espontaneismo con un 13 $\%$, modelo tecnológico con un $37 \%$ y al constructivismo con un $17 \%$, lo que significa que existe una apropiación débil del modelo que direcciona el Proyecto Institucional Educativo del establecimiento formativo donde realiza su quehacer profesional, dándole importancia al modelo tecnológico que inclusive de este podría tomar sus fortalezas para incorporarlas dentro del modelo constructivista.

En la verificación de las prácticas docentes constructivistas, se identificó mediante dos observaciones de clase y dos encuestas a estudiantes antes y después de la intervención en el aula con base en talleres que en las observaciones se evidenció una apropiación del constructivismo social con mayor fuerza, lo cual impactó a los estudiantes frente a los ambientes de clase, el entorno de aprendizaje, la interacción docente-estudiante, las estrategias metodológicas y la forma de evaluar, resultando así una experiencia significativa tanto para docentes como para estudiantes, donde se pudo superar algunas debilidades, no obstante aún se perciben insuficiencias en la apropiación de estrategias metodológicas y evaluación; por lo tanto, es importante una reflexión productiva que genere ajustes para transformar la manera de enseñar y propicie el aprendizaje activo, colaborativo, por proyectos, de tal manera que se movilice un verdadero intercambio del contexto social y cultural de los estudiantes para la construcción de significados a partir de su realidad.

Con respecto a la encuesta a estudiantes frente a la percepción de las prácticas docentes, se obtuvo que en el ambiente del aula del $74 \%$ subió al $93 \%$; en la norma de convivencia, del $83 \%$ al $95 \%$; en el entorno de aprendizaje, del $75 \%$ al $91 \%$; en la interacción docente-estudiante, del $83 \%$ al $95 \%$; en las estrategias metodológicas, del $76 \%$ al $89 \%$; y en la evaluación, del $72 \%$ al $87 \%$. En consecuencia, tuvo cierta afinidad con la observación ya que se evidenció que las debilidades estuvieron alineadas en cuanto a estrategias metodológicas y evaluación. 


\section{Referencias}

Bernal A. (2004). Educación, pedagogía y modelos pedagógicos. Editorial Paideia. $\mathrm{N}^{\circ}$ 11 2004. Editorial Mc Graw - Hill. p.5

Castillo, S. (2008). Propuesta pedagógica basada en el constructivismo para el uso óptimo de las tic en la enseñanza y el aprendizaje de la matemática. Revista Latinoamericana de Investigación en Matemática Educativa, 11 (2), 171-194

Díaz, A., \& Hernández, G. (1999). Estrategias docentes para un aprendizaje significativo. México: Editorial McGraw-Hill.

Corbetta, P. (2003). Metodología y técnicas de investigación social. Madrid. Mc Graw-Hill, pp.448

Elliot, J. (1993). La investigación-acción en educación. Editorial Morata.

Elliot, J. (2000). La investigación-acción en educación. Madrid: Editorial Morata.

Esteve, J. (2004). La tercera revolución educativa: la educación en la sociedad del conocimiento. Barcelona: Paidós Ibérica.

Flórez, R. (1994). Hacia una pedagogía del conocimiento. Editorial Mc-GrawHill.p.114-115.

Gardner, H. (1995). Inteligencias múltiples. La teoría en la práctica. Barcelona: Editorial Paidós.

Gómez, A., \& Perdomo, D. (2015). Las prácticas pedagógicas de los docentes de grado quinto de básica primaria de la institución educativa fundadores Ramón Bueno y José Triana, en relación con la implementación del modelo pedagógico constructivista (Tesis de Maestría). Universidad del Tolima, Ibagué, Tolima.

ICFES. (2016). Reporte de la Excelencia. Indicé Sintético de Calidad Educativa. Bogotá. UNIDEA.

Londoño, C. (2016). Constructivismo y teoría de la historia. Cuestiones De Filosofía, (16), 161-184. https://doi.org/10.19053/01235095.3954

MEN. (1994). Decreto 1860 de Agosto 3 de 1994. Diario oficial No. 41.473 del 5 de Agosto de 1994.

MEN (2006). Guía para el mejoramiento institucional. Copy Right.

MEN. (2008). Revolución Educativa. Diseño y ajustes para Proyecto Educativo Institucional. Bogotá. Mineducación.

Nieto, L. (2008). Instrumento para identificar modelos pedagógicos en el Instituto Técnico Rafael Reyes de la ciudad de Duitama.

Ñeco, Q. (1999). Rol del maestro en un esquema pedagógico constructivista. Bogotá. 
Nova, A. (2015). La formación integral: una apuesta de la educación superior. Cuestiones De Filosofía, 1(18), 185 - 214. https://doi.org/10.19053/01235095. v1.n18.2016.5363

Olson, J. (1982). Innovation in the science curriculum. London: Croom Helm.

Porlán, R. (1996). Constructivismo y escuela. Sevilla: Diada Editora.

Porlán, R. (1995).Hacia un modelo de enseñanza-aprendizaje basado en la investigación.

Sevilla. Diada Editora.

Piaget J. (1971). Psicología del niño. Décimo octava edición. Editorial Morata.

Pinedo, I., \& Yañez, J. (2017). La dimensión cognitiva de las emociones en la vida moral: los aportes de Martha Nussbauma al estado actual de la discusión. Cuestiones De Filosofía, 3(20), 105-127. https://doi.org/10.19053/01235095. v3.n20.2017.5919

Posner, G. (2005). Análisis del Currículo (3ª . Ed.). McGraw-Hill.

Ortiz. A. (2011).Hacia una nueva clasificación de los modelos pedagógicos: el pensamiento configuracional como paradigma científico y educativo del siglo XXI. Revista Praxis, 7, 121 - 137.

Vigotsky, L. (1978). Mind in society: the development of higher psychological process. Cambridge, MA: Harvard University Press. 